\title{
Hall Effect on Peristaltic Flow of Third Order Fluid in a Porous Medium with Heat and Mass Transfer
}

\author{
Nabil T. M. Eldabe, Ahmed Y. Ghaly, Sallam N. Sallam, Khaled Elagamy, Yasmeen M. Younis \\ Department of Mathematics, Faculty of Education, Ain Shams University, Cairo, Egypt \\ Email: Mohamed.unis@yahoo.com
}

Received 1 July 2015; accepted 15 September 2015; published 18 September 2015

Copyright (C) 2015 by authors and Scientific Research Publishing Inc.

This work is licensed under the Creative Commons Attribution International License (CC BY). http://creativecommons.org/licenses/by/4.0/

c) (i) Open Access

\section{Abstract}

We investigated the influence of hall, heat and mass transfer on the peristaltic flow of MHD third order fluid under long-wavelength and low Reynolds number approximation. The governing equations are solved analytically with the appropriate boundary conditions by using perturbation technique. The formula of velocity with temperature and concentration is obtained analytically as a function of the physical parameters of the problem.

\section{Keywords}

Peristaltic Flow, Third Order Fluid, Hall Effect, Heat, Mass Transfer

\section{Introduction}

Many fluids in biological system are transported by peristalsis. The word peristalsis stems from the Greek word peristaltikos, which means clasping and compressing. Physically, it means the mechanism for pumping fluid in a tube by means of a moving contractile ring around the tube, which pushes the material onward. The need for peristaltic pumping may arise in circumstances where it is desirable to avoid using any internal moving parts such as pistons to be one of the main mechanisms of fluid transport in a biological system. The application of peristaltic motion as a mean of transporting fluid has aroused interested in engineering fields. Latham [1] was probably the first to study the mechanism of peristaltic pumping in his M. S. Thesis. Several researches have analyzed the phenomenon of peristaltic transport under various assumptions. Haroun [2] studied the effect of a third-order fluid on the peristaltic transport in an asymmetric channel. In his study, the wavelength of the peristaltic waves is assumed to be large compared to the varying channel width, whereas the wave amplitudes need not be small compared to the varying channel width. Eldabe et al. [3] analyzed the incompressible flow of electrically con- 
ducting biviscosity fluid through an axisymmetric nonuniform tube with a sinusoidal wave under the considerations of long wavelength and low Reynolds number.

In the last years, several simple flow problems of classical hydrodynamics have received new attention in the more general context magnetohydrodynamics (MHD). The study of the motion of non-Newtonian fluids in the presense of the magnetic field has applications in many devices such as magneto hydrodynamic (MHD) power generator, MHD pumps, bioengineering devices and accelerators. Also it has been established that the biological systems are greatly affected by the application of the external magnetic field. Moreover, the MHD flow of a fluid in a channel with elastic, rhythmically contracting walls (peristaltic flow) is of interest in connection with certain problems of the movement of conductive physiological fluids. Some recent investigations made to discuss the mechanism of MHD include the works. Hayat et al. [4] studied the peristaltic transport of a third order fluid under the effect of a magnetic field. Srinivas and Kothandapani [5] have studied the influence of heat and mass transfer on MHD peristaltic flow through a porous space with compliant walls. Another important aspect in MHD is related to Hall effect. Such effect cannot be overlooked when flow subject to high magnetic field is considered. Siddiqui et al. [6] studied effects of Hall current and heat transfer on MHD flow of a Burgers fluid due to a pull of eccentric rotating disks. Hall effects on peristaltic flow of a Maxwell fluid in a porous medium have been studied by Hayat et al. [7] studied effects of Hall current and heat transfer on rotating flow of a second grade fluid through a porous medium. Khalid Nowar [8] studied Peristaltic Flow of a Nanofluid under the effect of Hall Current and Porous Medium.

The study of the influence of mass and heat transfer on non-Newtonian fluids has become important in the last few years. This importance is due to number of industrial processes. Examples are food processing, biochemical operations and transport in polymers, biomedical engineering; micro fabrication technologies etc., besides these biological tissues with heat transfer involve modes like heat conduction in tissues, heat convection by blood flow through the pores of tissue and radiation heat transfer between surface and its environment. Motivated by such facts, the peristaltic flow with heat transfer has been explored. El-Dabe et al. [9] studied magnetohydrodynamic flow and heat transfer for a peristaltic motion of carreau fluid through a porous medium. El-Dabe et al. [10] studied Peristaltic Motion of Non-Newtonian Fluid with Heat and Mass Transfer through a Porous Medium in Channel under Uniform Magnetic Field. El-Dabe et al. [11] analyzed the Magnetohydrodynamic Peristaltic motion with heat and mass transfer of a Jeffery fluid in a tube through porous medium.

With the above discussion in mind, we propose to study the peristaltic motion of non-Newtonian fluid through a porous medium in the channel under the effect of magnetic field. A third order non-Newtonian constitutive model is employed for the transport fluid. The effects of hall, body temperature and concentration are taken into consideration. The governing equations of motion, energy, and concentration have been reduced under the assumption of long wavelength. The reduced equations are then solved analytically via perturbation method. The physical behaviors of emerging parameters are discussed through graphs.

\section{Mathematical Analysis}

Consider a two-dimensional channel of uniform thickness $2 a$, filled with incompressible homogeneous electrically conducting non-Newtonian third order fluid through a porous medium with heat and mass transfer. The channels walls are considered and flexible the vertical displacements for the upper and lower walls are $H$ and $-H$, see Figure 1, where $H$ is defined by

$$
H^{*}\left(X^{*}, t^{*}\right)=a+b \sin \frac{2 \pi}{\lambda}\left(X^{*}-c t^{*}\right)
$$

where In the above equation $b$ is the wave amplitude, $\lambda$ is the wave length and $t^{*}$ is the time. A uniform magnetic field with magnetic flux density vector $B=\left(0,0, B_{0}\right)$ is applied, neglecting the induced magnetic field under the assumption that the magnetic Reynolds number is small, the expression for the current density $\underline{J}$ including the Hall effect and neglecting ion-slip and thermoelectric effects is given by

$$
\underline{J}=\sigma\left[\underline{E}+\underline{V}^{*} \wedge \underline{B}-\frac{1}{e n_{e}}(\underline{J} \wedge \underline{B})\right],
$$

where $\sigma$ is the electric conductivity of the fluid is, $\underline{V}^{*}$ is the velocity vector, It is also assumed that $\underline{E}=0$ 


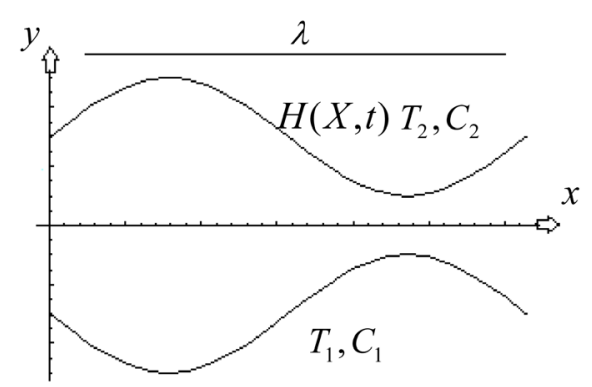

Figure 1. Sketch of the problem.

(since there is no applied polarization voltage), $m=\frac{\sigma B_{0}}{e n_{e}}$ is the Hall parameter, $e$ is the electric charge and $n_{e}$ is the number of density of electron. The constitutive equation for the non-Newtonian third order fluid can be written as in [4].

Consider

$$
\begin{gathered}
\tau^{*}=-P^{*} I+S^{*}, \\
S^{*}=\mu A_{1}^{*}+\alpha_{1} A_{2}^{*}+\alpha_{2} A_{1}^{* 2}+\beta\left(\operatorname{tr} A_{1}^{* 2}\right) A_{1}^{*} .
\end{gathered}
$$

Here $\underline{\tau}^{*}$ is the extra stress tensor, $-P^{*} I$ is the indeterminate part of the stress due to the constraint of incompressibility and $A_{n}^{*}$ are the Rivlin-Ericksen tensors, defined by

$$
\begin{aligned}
& A_{1}^{*}=\left(\operatorname{grad} \underline{V}^{*}\right)+\left(\operatorname{grad} \underline{V}^{*}\right)^{\mathrm{T}}, \\
& A_{n}^{*}=\frac{\mathrm{d} A_{n-1}^{*}}{\mathrm{~d} t^{*}}+A_{n-1}^{*}\left(\operatorname{grad} \underline{V}^{*}\right)+\left(\operatorname{grad} \underline{V}^{*}\right)^{\mathrm{T}} A_{n-1}^{*}, \quad n>1,
\end{aligned}
$$

where grad denotes the gradient operator, $\frac{\mathrm{d}}{\mathrm{d} t^{*}}$ the material time derivative, $\mu$ is the coefficient of shear viscosity, the normal stress coefficients $\alpha_{1}$ and $\alpha_{2}$, and the coefficient $\beta$,

$$
\mu \geq 0, \alpha_{1} \geq 0, \alpha_{2} \geq 0, \beta \geq 0, \quad\left|\alpha_{1}+\alpha_{2}\right| \leq \sqrt{24 \mu \beta}
$$

The fundamental equations governing this model together with the generalized Ohm's law taking the effects of Hall currents and Maxwell's equations into account are

$$
\begin{gathered}
\nabla \cdot \underline{V}^{*}=0, \\
\rho \frac{\mathrm{d} \underline{V}^{*}}{\mathrm{~d} t^{*}}=-\nabla P^{*}+\nabla \cdot \underline{\tau}^{*}+\underline{J} \wedge \underline{B}, \\
\rho c_{p}\left[\frac{\partial T}{\partial t^{*}}+\left(\underline{V}^{*} \cdot \nabla\right) T\right]=\kappa \nabla^{2} T+\Phi+\nabla \cdot q_{r}, \\
{\left[\frac{\partial C}{\partial t^{*}}+\left(\underline{V}^{*} \cdot \nabla\right) C\right]=D \nabla^{2} C+\frac{D \kappa_{T}}{T_{m}} \nabla^{2} T-L_{c}\left(C-C_{2}\right) .}
\end{gathered}
$$

where $\rho$ is the density of the fluid is, $P^{*}$ is the pressure, $c_{p}$ is the specific heat capacity at constant pressure, $T$ is the temperature, $\kappa$ is the thermal conductivity, $\Phi$ is the dissipation function, $q_{r}$ is the radiative heat flux, $C$ is the concentration of the fluid, $D$ is the coefficient of mass diffusivity, $\kappa_{T}$ is the thermal diffusion ratio, $T_{m}$ is the mean fluid temperature and $L_{c}$ is the reaction rate constant.

By using Rosselant approximation we have

$$
q_{Y}=\frac{4 \sigma_{0}}{3 K_{0}} \frac{\partial T^{4}}{\partial Y} .
$$


where $\sigma_{0}$ is the Stefan Boltizman constant and $K_{0}$ is the mean absorption coefficient. We assume that the temperature differences within the flow are sufficiently small such that $T^{4}$ may be expressed as a linear function of temperature. This is accomplished by expanding $T^{4}$ in a Taylor series about $T_{2}$, and neglecting higher order terms, we get

$$
T^{4} \approx 4 T_{2}^{3} T-3 T_{2}^{4} .
$$

The equations governing the two-dimensional motion of this model (7)-(10)

$$
\begin{gathered}
\frac{\partial U^{*}}{\partial X^{*}}+\frac{\partial V^{*}}{\partial Y^{*}}=0, \\
\rho\left(\frac{\partial}{\partial t^{*}}+U^{*} \frac{\partial}{\partial X^{*}}+V^{*} \frac{\partial}{\partial Y^{*}}\right) U^{*}=-\frac{\partial P^{*}}{\partial X^{*}}+\frac{\partial S_{X^{*} X^{*}}^{*}}{\partial X^{*}}+\frac{\partial S_{X^{*} Y^{*}}^{*}}{\partial Y^{*}}-\frac{\mu}{k} U^{*}+\frac{\sigma B_{0}^{2}}{1+m^{2}}\left(m V^{*}-U^{*}\right), \\
\rho\left(\frac{\partial}{\partial t^{*}}+U^{*} \frac{\partial}{\partial X^{*}}+V^{*} \frac{\partial}{\partial Y^{*}}\right) V^{*}=-\frac{\partial P^{*}}{\partial Y^{*}}+\frac{\partial S_{X^{*} Y^{*}}^{*}}{\partial X^{*}}+\frac{\partial S_{Y^{*} Y^{*}}^{*}}{\partial Y^{*}}-\frac{\mu}{k} V^{*}-\frac{\sigma B_{0}^{2}}{1+m^{2}}\left(m U^{*}+V^{*}\right),
\end{gathered}
$$

where

$$
\begin{gathered}
S_{X^{*} X^{*}}^{*}=2 \mu U_{X^{*}}^{*}+\alpha_{1}\left(2 U_{t X}+2 U U_{X X}+2 V U_{X^{*} Y^{*}}^{*}+4 U_{X^{*}}^{* 2}+2 V_{X^{*}}^{*} U_{Y^{*}}^{*}+2 U_{Y^{*}}^{* 2}\right) \\
+\alpha_{2}\left(4 U_{X^{*}}^{* 2}+2 V_{X^{*}}^{*} U_{Y^{*}}^{*}+U_{Y^{*}}^{* 2}+V_{X^{*}}^{* 2}\right)+2 \beta\left(U_{X^{*}}^{* 3}+2 U_{X^{*}}^{*} V_{X^{*}}^{* 2}+2 U_{X^{*}}^{*} U_{Y^{*}}^{* 2}+2 V_{X^{*}}^{*} U_{X^{*}}^{*} U_{Y^{*}}^{*}+4 U_{X^{*}}^{*} V_{Y^{*}}^{* 2}\right), \\
S_{X^{*} Y^{*}}^{*}=\mu\left(U_{Y^{*}}^{*}+V_{X^{*}}^{*}\right)+\alpha_{1}\left(V_{X^{*} t^{*}}^{*}+U_{t^{*} Y^{*}}^{*}+U U_{X^{*} X^{*}}^{*}+U V_{X^{*} X^{*}}^{*}+V U_{Y^{*} Y^{*}}^{*}+V V_{X^{*} Y^{*}}^{*}+2 U_{X^{*}}^{*} U_{Y^{*}}^{*}+2 V_{X^{*}}^{*} V_{Y^{*}}^{*}\right. \\
+\alpha_{2}\left(2 U_{X^{*}}^{*} U_{Y^{*}}^{*}+2 V_{X^{*}}^{*} V_{Y^{*}}^{*}\right)+2 \beta\left(U_{X^{*}}^{* 2} U_{Y^{*}}^{*}+2 U_{Y^{*}}^{* 3}+6 V_{X^{*}}^{* 2} U_{Y^{*}}^{*}+4 V_{Y^{*}}^{* 2} U_{Y^{*}}^{*}+6 V_{X^{*}}^{*} U_{Y^{*}}^{* 2}+V_{X^{*}}^{*} U_{X^{*}}^{* 2}+2 V_{X^{*}}^{* 3}+4 V_{Y^{*}}^{* 2} V_{X^{*}}^{*}\right),
\end{gathered}
$$

and

$$
\begin{aligned}
& S_{Y^{*} Y^{*}}^{*}=2 \mu V_{Y^{*}}^{*}+\alpha_{1}\left(2 V_{t Y^{*}}^{*}+2 U V_{X^{*} Y^{*}}^{*}+2 V V_{Y^{*} Y^{*}}^{*}+2 U_{Y^{*}}^{* 2}+2 V_{X^{*}}^{*} U_{Y^{*}}^{*}+4 V_{Y^{*}}^{* 2}\right) \\
& +\alpha_{2}\left(U_{Y^{*}}^{* 2}+2 V_{X^{*}}^{*} U_{Y^{*}}^{*}+4 V_{Y^{*}}^{* 2}+V_{X^{*}}^{* 2}\right)+2 \beta\left(4 V_{Y^{*}}^{* 3}+U V_{Y^{*}}^{*}+2 V_{X^{*}}^{* 2} V_{Y}+2 V_{X^{*}}^{*} U_{X^{*}}^{*} U_{Y^{*}}^{*}+4 U_{Y^{*}}^{* 2} V_{Y^{*}}^{*}\right),
\end{aligned}
$$

where $\left(U^{*}, V^{*}\right)$ is the velocity components in fixed frame of reference $\left(X^{*}, Y^{*}\right)$

The dissipation function $\Phi$ can be written as follows

$$
\begin{gathered}
\Phi=\tau_{i j}^{*} \frac{\partial V_{i}^{*}}{\partial X_{j}^{*}}, \\
\Phi=S_{X^{*} X^{*}}^{*} \frac{\partial U^{*}}{\partial X^{*}}+S_{X^{*} Y^{*}}^{*}\left(\frac{\partial V^{*}}{\partial X^{*}}+\frac{\partial U^{*}}{\partial Y^{*}}\right)+S_{Y^{*} Y^{*}}^{*} \frac{\partial V^{*}}{\partial Y^{*}} \\
\rho C_{p}\left[\frac{\partial T}{\partial t^{*}}+U^{*} \frac{\partial T}{\partial X^{*}}+V^{*} \frac{\partial T}{\partial Y^{*}}\right] \\
=\kappa\left(\frac{\partial^{2} T}{\partial X^{* 2}}+\frac{\partial^{2} T}{\partial Y^{* 2}}\right)+S_{X^{*} X^{*}}^{*} \frac{\partial U^{*}}{\partial X^{*}}+S_{X^{*} Y^{*}}^{*}\left(\frac{\partial V^{*}}{\partial X^{*}}+\frac{\partial U^{*}}{\partial Y^{*}}\right)+S_{Y^{*} Y^{*}}^{*} \frac{\partial V^{*}}{\partial Y^{*}}+\frac{16 \sigma_{0}}{3 K_{0}} T_{2}^{3} \frac{\partial^{2} T}{\partial Y^{* 2}}, \\
{\left[\frac{\partial C}{\partial t^{*}}+U^{*} \frac{\partial C}{\partial X^{*}}+V^{*} \frac{\partial C}{\partial Y^{*}}\right]=D\left(\frac{\partial^{2} C}{\partial X^{* 2}}+\frac{\partial^{2} C}{\partial Y^{* 2}}\right)+\frac{D \kappa_{T}}{T_{m}}\left(\frac{\partial^{2} T}{\partial X^{* 2}}+\frac{\partial^{2} T}{\partial Y^{* 2}}\right)-L_{c}\left(C-C_{2}\right),}
\end{gathered}
$$

The appropriate boundary conditions taken as follows:

$$
\begin{aligned}
& U^{*}=0, T=T_{2}, C=C_{2} \quad \text { at } Y^{*}=H^{*}, \\
& U^{*}=0, T=T_{1}, C=C_{1} \quad \text { at } Y^{*}=-H^{*},
\end{aligned}
$$

Consider a wave frame $\left(x^{*}, y^{*}\right)$ which moving with speed $c$. Coordinates and velocity components in wave frame are related by the following transformations 


$$
x^{*}=X^{*}-c t, y^{*}=Y^{*}, u^{*}=U^{*}-c, v^{*}=V^{*}, p^{*}\left(x^{*}, y^{*}\right)=P^{*}\left(X^{*}, Y^{*}, t\right)
$$

In which $\left(u^{*}, v^{*}\right)$ are components of the velocity in the moving coordinates system.

Then, the system of Equations (12)-(22) can be written as:

$$
\begin{gathered}
\frac{\partial u^{*}}{\partial x^{*}}+\frac{\partial v^{*}}{\partial y^{*}}=0, \\
\rho\left(u^{*} \frac{\partial}{\partial x^{*}}+v^{*} \frac{\partial}{\partial y^{*}}\right) u^{*}=-\frac{\partial p^{*}}{\partial x^{*}}+\frac{\partial s_{x^{*} x^{*}}^{*}}{\partial x^{*}}+\frac{\partial s_{x^{*} y^{*}}}{\partial y^{*}}-\frac{\mu}{k} u^{*}+\frac{\sigma B_{0}^{2}}{1+m^{2}}\left(m v^{*}-u^{*}\right), \\
\rho\left(u^{*} \frac{\partial}{\partial x^{*}}+v^{*} \frac{\partial}{\partial y^{*}}\right) v^{*}=-\frac{\partial P}{\partial y^{*}}+\frac{\partial s_{x^{*} y^{*}}^{*}}{\partial x^{*}}+\frac{\partial s_{y^{*} y^{*}}^{*}}{\partial y^{*}}-\frac{\mu}{k} v^{*}-\frac{\sigma B_{0}^{2}}{1+m^{2}}\left(m u^{*}+v^{*}\right),
\end{gathered}
$$

where

$$
\begin{aligned}
& s_{x^{*} x^{*}}^{*}=2 \mu u_{x^{*}}^{*}+\alpha_{1}\left(2 u^{*} u_{x^{*} x^{*}}^{*}+2 v^{*} u_{x^{*} y^{*}}^{*}+4 u_{x^{*}}^{* 2}+2 v_{x^{*}}^{*} u_{y^{*}}^{*}+2 u_{y^{*}}^{* 2}\right)+\alpha_{2}\left(4 u_{x^{*}}^{* 2}+2 v_{x^{*}}^{*} u_{y^{*}}^{*}+u_{y^{*}}^{* 2}+v_{x^{*}}^{* 2}\right) \\
& +2 \beta\left(u_{x^{*}}^{* 3}+2 u_{x^{*}}^{*} v_{x^{*}}^{* 2}+2 u_{x^{*}}^{*} u_{y^{*}}^{* 2}+2 v_{x^{*}}^{*} u_{x^{*}}^{*} u_{y^{*}}^{*}+4 u_{x^{*}}^{*} v_{y^{*}}^{* 2}\right) \text {, } \\
& s_{x^{*} y^{*}}^{*}=\mu\left(u_{y^{*}}^{*}+v_{x^{*}}^{*}\right)+\alpha_{1}\left(u^{*} u_{x^{*} x^{*}}^{*}+u^{*} v_{x^{*} x^{*}}^{*}+v^{*} u_{y^{*} y^{*}}^{*}+v^{*} v_{x^{*} y^{*}}^{*}+2 u_{x^{*}}^{*} u_{y^{*}}^{*}+2 v_{x^{*}}^{*} v_{y^{*}}^{*}\right)+\alpha_{2}\left(2 u_{x^{*}}^{*} u_{y^{*}}^{*}+2 v_{x^{*}}^{*} v_{y^{*}}^{*} v^{*}\right) \\
& +2 \beta\left(u_{x^{*}}^{* 2} u_{y^{*}}^{*}+2 u_{y^{*}}^{* 3}+6 v_{x^{*}}^{* 2} u_{y^{*}}^{*}+4 v_{y^{*}}^{* 2} u_{y^{*}}^{*}+6 v_{x^{*}}^{*} u_{y^{*}}^{* 2}+v_{x^{*}}^{*} u_{x^{*}}^{* 2}+2 v_{x^{*}}^{* 3}+4 v_{y^{*}}^{* 2} *_{x^{*}}^{*}\right) \text {, }
\end{aligned}
$$

And

$$
\begin{aligned}
& s_{y^{*} y^{*}}^{*}= 2 \mu v_{y^{*}}^{*}+\alpha_{1}\left(2 u^{*} v_{x^{*} y^{*}}^{*}+2 v^{*} v_{y^{*} y^{*}}^{*}+2 u_{y^{*}}^{*}+2 v_{x^{*}}^{*} u_{y^{*}}^{*}+4 v_{y^{*}}^{* 2}\right)+\alpha_{2}\left(u_{y^{*}}^{* 2}+2 v_{x^{*}}^{*} u_{y^{*}}^{*}+4 v_{y^{*}}^{* 2}+v_{x^{*}}^{* 2}\right) \\
&+2 \beta\left(4 v_{y^{*}}^{* 3}+u_{x^{*}}^{* 2} v_{y^{*}}^{*}+2 v_{x^{*}}^{*} v_{y^{*}}^{*}+2 v_{x^{*}}^{*} u_{x^{*}}^{*} u_{y^{*}}^{*}+4 u_{y^{*}}^{* 2} v_{y^{*}}^{*}\right), \\
& \rho c_{p}\left[u^{*} \frac{\partial T}{\partial x^{*}}+v^{*} \frac{\partial T}{\partial y^{*}}\right]=\kappa\left(\frac{\partial^{2} T}{\partial x^{* 2}}+\frac{\partial^{2} T}{\partial y^{* 2}}\right)+s_{x^{*} x^{*}}^{*} \frac{\partial u^{*}}{\partial x^{*}}+s_{x^{*} y^{*}}^{*}\left(\frac{\partial v^{*}}{\partial x^{*}}+\frac{\partial u^{*}}{\partial y^{*}}\right)+s_{y^{*} y^{*}}^{*} \frac{\partial v^{*}}{\partial y^{*}}+\frac{16 \sigma_{0}}{3 K_{0}} T_{2}^{3} \frac{\partial^{2} T}{\partial y^{* 2}}, \\
& {\left[u^{*} \frac{\partial C}{\partial x^{*}}+v^{*} \frac{\partial C}{\partial y^{*}}\right]=D\left(\frac{\partial^{2} C}{\partial x^{* 2}}+\frac{\partial^{2} C}{\partial y^{* 2}}\right)+\frac{D \kappa_{T}}{T_{m}}\left(\frac{\partial^{2} T}{\partial x^{* 2}}+\frac{\partial^{2} T}{\partial y^{* 2}}\right)-L_{c}\left(C-C_{2}\right), }
\end{aligned}
$$

The boundary conditions become:

$$
\begin{aligned}
& u^{*}=-C, T=T_{2}, C=C_{2} \quad \text { at } y^{*}=H, \\
& u^{*}=-C, T=T_{1}, C=C_{1} \quad \text { at } y^{*}=-H,
\end{aligned}
$$

We introduce the following non-dimensional quantities:

$$
\begin{aligned}
& x=\frac{x^{*}}{\lambda}, \quad y=\frac{y^{*}}{a}, \quad u=\frac{u^{*}}{c}, \quad v=\frac{v^{*}}{c \delta}, \quad p=\frac{a^{2} p^{*}}{\lambda \mu c}, \\
& \delta=\frac{2 \pi a}{\lambda}, \quad R_{e}=\frac{\rho c a}{\mu}, \quad h=\frac{H}{a}, \quad S=\frac{a}{\mu c} S^{*}, \quad \theta=\frac{T-T_{2}}{T_{1}-T_{2}}, \quad \phi=\frac{C-C_{2}}{C_{1}-C_{2}}, \\
& D_{a}=\frac{k}{a^{2}}, \quad M=\sqrt{\frac{\sigma}{\mu}} B_{0} a, \quad \lambda_{1}=\frac{\alpha_{1} c}{\mu a}, \quad \lambda_{2}=\frac{\alpha_{2} c}{\mu a}, \quad \Gamma=\frac{\beta c^{2}}{\mu a^{2}}, \\
& P_{r}=\frac{\mu c_{p}}{\kappa}, \quad E_{c}=\frac{c^{2}}{c_{p}\left(T_{1}-T_{0}\right)}, \quad R_{n}=\frac{\mu c_{p} K_{0}}{4 \sigma_{0} T_{2}^{3}}, \quad S_{c}=\frac{\mu}{\rho D}, \\
& S_{r}=\frac{\rho D K_{T}\left(T_{1}-T_{0}\right)}{T_{m} \mu\left(C_{1}-C_{0}\right)}, \quad R_{C}=\frac{\rho a^{2} L_{c}\left(C_{1}-C_{2}\right)}{\mu} .
\end{aligned}
$$


where the non-dimensional wave number $\delta$, the Reynolds number $R_{e}$, the material coefficients are $\left(\lambda_{1}, \lambda_{2}\right)$, Deborh number is $\Gamma$, Darcy number is $D_{a}, M$ is the Hartman number, $P_{r}$ is the Prandtl number, $E_{c}$ is the Eckert number, $R_{n}$ is the Radiation parameter, $S_{c}$ is the Schmidt number, $S_{r}$ is the Soret number and $R_{C}$ is the Chemical reaction parameter.

Substituting (33) into Equations (24)-(32) we obtain the following non-dimensional equations:

$$
\begin{gathered}
\frac{\partial u}{\partial x}+\frac{\partial v}{\partial y}=0, \\
\delta \operatorname{Re}\left(u \frac{\partial}{\partial x}+v \frac{\partial}{\partial y}\right) u=-\frac{\partial p}{\partial x}+\frac{\partial s_{x x}}{\partial x}+\delta \frac{\partial s_{x y}}{\partial y}-\frac{1}{D_{a}}(u+1)+\frac{M}{1+m^{2}}(m \delta v-(u+1)), \\
\delta^{3} \operatorname{Re}\left(u \frac{\partial}{\partial x}+v \frac{\partial}{\partial y}\right) v=-\frac{\partial p}{\partial y}+\delta^{2} \frac{\partial s_{x y}}{\partial x}+\delta \frac{\partial s_{y y}}{\partial y}-\frac{\delta^{2}}{D_{a}} v-\frac{\delta M}{1+m^{2}}(m(u+1)+\delta v),
\end{gathered}
$$

where

$$
\begin{aligned}
s_{x x}= & 2 \delta u_{x}+\lambda_{1}\left(2 \delta^{2} u u_{x x}+2 \delta^{3} v u_{x y}+4 \delta^{2} u_{x}^{2}+2 \delta v_{x} u_{y}+2 \delta^{3} v_{x}^{2}\right)+\lambda_{2}\left(4 \delta^{2} u_{x}^{2}+2 \delta^{2} v_{x} u_{y}+u_{y}^{2}+\delta^{4} v_{x}^{2}\right) \\
& +2 \Gamma\left(\delta^{3} u_{x}^{3}+2 \delta^{5} u_{x} v_{x}^{2}+2 \delta u_{x} u_{y}^{2}+2 \delta^{3} v_{x} u_{x} u_{y}+4 \delta u_{x} v_{y}^{2}\right), \\
s_{x y}= & \left(u_{y}+\delta^{2} v_{x}\right)+\lambda_{1}\left(\delta^{2} u u_{x x}+\delta u v_{x x}+\delta v u_{y y}+\delta^{3} v v_{x y}+2 \delta u_{x} u_{y}+2 \delta^{3} v_{x} v_{y}\right)+\lambda_{2}\left(2 \delta^{2} u_{x} u_{y}+2 \delta^{3} v_{x} v_{y}\right) \\
& +2 \Gamma\left(\delta^{2} u_{x}^{2} u_{y}+2 u_{y}^{3}+6 \delta^{4} v_{x}^{2} u_{y}+4 \delta^{2} v_{y}^{2} u_{y}+6 \delta^{2} v_{x} u_{y}^{2}+\delta^{4} v_{x} u_{x}^{2}+2 \delta^{3} v_{x}^{3}+4 \delta^{3} v_{y}^{2} v_{x}\right),
\end{aligned}
$$

and

$$
\begin{gathered}
s_{y y}=2 \delta v_{y}+\lambda_{1}\left(2 \delta^{2} u v_{x y}+2 \delta^{2} v v_{y y}+2 u_{y}^{2}+2 \delta^{2} v_{x} u_{y}+4 \delta^{2} v_{y}^{2}\right)+\lambda_{2}\left(u_{y}^{2}+2 \delta^{2} v_{x} u_{y}+4 \delta^{2} v_{y}^{2}+\delta^{4} v_{x}^{2}\right) \\
+2 \Gamma\left(4 \delta^{3} v_{y}^{3}+\delta^{2} u_{x}^{2} v_{y}+2 \delta^{5} v_{x}^{2} v_{y}+2 \delta^{3} v_{x} u_{x} u_{y}+4 \delta u_{y}^{2} v_{y}\right), \\
\delta \operatorname{Re}\left[u \frac{\partial \theta}{\partial x}+v \frac{\partial \theta}{\partial y}\right]=\frac{1}{P_{r}}\left(\delta^{2} \frac{\partial^{2} \theta}{\partial x^{2}}+\frac{\partial^{2} \theta}{\partial y^{2}}\right)+E_{c} \delta s_{x x} \frac{\partial u}{\partial x}+E_{c} s_{x y}\left(\delta \frac{\partial v}{\partial x}-\frac{\partial u}{\partial y}\right)+E_{c} s_{y y} \frac{\partial v}{\partial y}+\frac{4}{3 R_{n}} \frac{\partial^{2} \theta}{\partial y^{2}}, \\
\delta \operatorname{Re}\left[u \frac{\partial C}{\partial x}+v \frac{\partial C}{\partial y}\right]=\frac{1}{S_{c}}\left(\delta^{2} \frac{\partial^{2} \phi}{\partial x^{2}}+\frac{\partial^{2} \phi}{\partial y^{2}}\right)+S_{r}\left(\delta^{2} \frac{\partial^{2} \theta}{\partial x^{2}}+\frac{\partial^{2} \theta}{\partial y^{2}}\right)-R_{c} \phi
\end{gathered}
$$

With conditions:

$$
\begin{aligned}
& u=-1, \quad \theta=0, \phi=0 \quad \text { at } y=h, \\
& u=-1, \quad \theta=1, \phi=1 \quad \text { at } y=-h,
\end{aligned}
$$

We also note that $h$ represents the dimensionless form of the surface of the peristaltic wall.

$$
h=1+\chi \sin x
$$

where, $\chi=\frac{b}{a}$ is the amplitude ratio or the occlusion under the assumptions of long wavelength $(\delta \ll 1)$. The Equations (35)-(42) take the following form:

$$
\begin{gathered}
\frac{\partial p}{\partial x}=\frac{\partial s_{x y}}{\partial y}-\left(\frac{1}{D_{a}}+\frac{M}{1+m^{2}}\right)(u+1), \\
\frac{\partial p}{\partial y}=0 \\
s_{x x}=\left(2 \lambda_{1}+\lambda_{2}\right) u_{y}^{2} \\
s_{x y}=\frac{\partial u}{\partial y}+2 \Gamma\left(\frac{\partial u}{\partial y}\right)^{3}
\end{gathered}
$$




$$
\begin{gathered}
S_{y y}=\left(2 \lambda_{1}+\lambda_{2}\right) u_{y}^{2}, \\
\left(\frac{1}{P_{r}}+\frac{4}{3 R_{n}}\right) \frac{\partial^{2} \theta}{\partial y^{2}}+E_{c} s_{x y} \frac{\partial u}{\partial y}=0, \\
\frac{\partial^{2} \phi}{\partial y^{2}}-S_{c} R_{c} \phi=-S_{c} S_{r} \frac{\partial^{2} \theta}{\partial y^{2}} .
\end{gathered}
$$

Eliminating $p$ from Equations (44) and (45), we have the following equation

$$
\frac{\partial^{2} s_{x y}}{\partial y^{2}}-\left(\frac{1}{D_{a}}+\frac{M}{1+m^{2}}\right) \frac{\partial u}{\partial y}=0,
$$

$\frac{\partial S_{x y}}{\partial y}-\left(\frac{1}{D_{a}}+\frac{M}{1+m^{2}}\right) u=A$, where $A$ is a constant.

\section{Series Solution}

For perturbation solution we write

$$
\begin{aligned}
& u=u_{0}+\Gamma u_{1}, \\
& \theta=\theta_{0}+\Gamma \theta_{1}, \\
& \phi=\varphi_{0}+\Gamma \phi_{1} .
\end{aligned}
$$

Substituting (52) in the Equations (45)-(49), equating the coefficients of like powers of $\Gamma$, we get the following

Zeroth order system:

$$
\begin{gathered}
\frac{\partial^{2} u_{0}}{\partial y^{2}}-N^{2} u_{0}=A, \\
\frac{\partial^{2} \theta_{0}}{\partial y^{2}}+E_{c} G\left(\frac{\partial u_{0}}{\partial y}\right)^{2}=0, \\
\frac{\partial^{2} \phi_{0}}{\partial y^{2}}-S_{c} R_{c} \phi_{0}=-S_{c} S_{r} \frac{\partial^{2} \theta_{0}}{\partial y^{2}},
\end{gathered}
$$

The subjected boundary conditions are:

$$
\begin{aligned}
& u_{0}=-1, \theta_{0}=0, \phi_{0}=0 \text { at } y=h, \\
& u_{0}=-1, \theta_{0}=1, \phi_{0}=1 \text { at } y=-h,
\end{aligned}
$$

First order system

$$
\begin{gathered}
\frac{\partial^{2} u_{1}}{\partial y^{2}}+2 \frac{\partial}{\partial y}\left(\frac{\partial u_{0}}{\partial y}\right)^{3}-N^{2} u_{1}=0, \\
\frac{\partial^{2} \theta_{1}}{\partial y^{2}}+E_{c} G\left[\left(\frac{\partial u_{1}}{\partial y}\right)^{2}+2\left(\frac{\partial u_{0}}{\partial y}\right)^{4}\right]=0, \\
\frac{\partial^{2} \phi_{1}}{\partial y^{2}}-S_{c} R_{c} \phi_{1}=-S_{c} S_{r} \frac{\partial^{2} \theta_{1}}{\partial y^{2}}, \\
u_{1}=0, \theta_{1}=0, \phi_{1}=0 \quad \text { at } y=h, \\
u_{1}=0, \theta_{1}=0, \phi_{1}=0 \quad \text { at } y=-h .
\end{gathered}
$$

The solution of zero order system can be obtained analytically as 


$$
\begin{gathered}
u_{0}=W_{1} \operatorname{Cosh} N y-\frac{A}{N^{2}}, \\
\theta_{0}=-\frac{E_{c} G N^{2} W_{1}^{2}}{4}\left(\frac{\operatorname{Cosh} 2 N y}{2 N^{2}}-y^{2}\right)-\frac{y}{2 h}+B_{1} \\
\phi_{0}=2 C_{1} \operatorname{Cosh} \sqrt{S_{c} R_{c}} y+\frac{e^{-\sqrt{S_{c} R_{c}} y}}{2 \operatorname{Sinh} \sqrt{S_{c} R_{c}} h}+\frac{S_{c} S_{r} E_{c} G N^{2} W_{1}^{2}}{2}\left(\frac{\operatorname{Cosh} 2 N y}{4 N^{2}-S_{c} R_{c}}+\frac{1}{S_{c} R_{c}}\right) .
\end{gathered}
$$

Also, the solution of first order system can be obtained analytically as

$$
\begin{gathered}
u_{1}=2 W_{2} \operatorname{Cosh} N y-\frac{3}{4} N^{4} W_{1}^{3}\left(\frac{\operatorname{Cosh} 3 N y}{4 N^{2}}-\frac{y}{N} \operatorname{Sinh} N y\right), \\
\theta_{1}=-E_{c} G\left(W_{1} W_{2} N^{2}\left(\frac{\operatorname{Cosh} 2 N y}{2 N^{2}}-y^{2}\right)-\frac{3}{2} N^{5} W_{1}^{4}\left(\frac{3}{16 N^{3}} \frac{\operatorname{Cosh} 4 N y}{8}-\frac{y \operatorname{Sinh} 2 N y}{8 N^{2}}-\frac{3 \operatorname{Cosh} 2 N y}{32 N^{3}}+\frac{y^{2}}{4 N}\right)\right. \\
\left.+\frac{N^{4} W_{1}^{4}}{8}\left(\frac{\operatorname{Cosh} 4 N y}{8 N^{2}}-\frac{2 \operatorname{Cosh} 2 N y}{N^{2}}+3 y^{2}\right)\right)+B_{2}, \\
\phi_{1}=S_{c} S_{r} E_{c} G\left(W_{1} W_{2} N^{2}\left(\frac{\operatorname{Cosh} 2 N y}{2 N^{2}}-y^{2}\right)-\frac{3 N^{5} W_{1}^{4}}{2}\left(\frac{3}{8 N^{3}} \frac{\operatorname{Cosh} 4 N y}{16}-\frac{y \operatorname{Sinh} 2 N y}{N}\right.\right. \\
\left.\left.+\left(\frac{1}{2 N^{2}}+\frac{11}{32 N^{3}}\right) \operatorname{Cosh} 2 N y-\frac{y^{2}}{2 N}\right)\right)+\frac{N^{4} W_{1}^{4}}{4}\left(\frac{\operatorname{Cosh} 4 N y}{16 N^{2}}-\frac{\operatorname{Cosh} 2 N y}{N^{2}}+\frac{3}{2} y^{2}\right)+C_{2} .
\end{gathered}
$$

where

$$
\begin{gathered}
N^{2}=\frac{1}{D_{a}}+\frac{M}{1+m^{2}}, \\
W_{1}=\frac{1}{\operatorname{Cosh} N h}\left(\frac{A}{N^{2}}-1\right), \\
W_{2}=\frac{3 N^{4} W_{1}^{3}}{8 \operatorname{Cosh} h h}\left(\frac{\operatorname{Cosh} 3 N h}{4 N^{2}}-\frac{h}{N} \operatorname{Sinh} N h\right), \\
B_{1}=\frac{E_{c} G N^{2} W_{1}^{2}}{4}\left(\frac{\operatorname{Cosh} 2 N h}{2 N^{2}}-h^{2}\right)+\frac{1}{2}, \\
B_{2}=E_{c} G\left(W_{1} W_{2} N^{2}\left(\frac{\operatorname{Cosh} 2 N h}{2 N^{2}-h^{2}}\right)-\frac{3}{2} N^{5} W_{1}^{4}\left(\frac{3}{16 N^{3}} \frac{\operatorname{Cosh} 4 N h}{8}-\frac{h \operatorname{Sinh} 2 N h}{8 N^{2}}\right.\right. \\
\left.\left.-\frac{3 \operatorname{Cosh} 2 N h}{32 N^{3}}+\frac{h^{2}}{4 N}\right)+\frac{N^{4} W_{1}^{4}}{8}\left(\frac{\operatorname{Cosh} 4 N h}{8 N^{2}}-\frac{2 \operatorname{Cosh} 2 N h}{N^{2}}+3 h^{2}\right)\right), \\
C_{1}=\frac{-1}{2 \operatorname{Cosh} \sqrt{S_{c} R_{c}} h}\left(\frac{\mathrm{e}^{-\sqrt{S_{c} R_{c}} h}}{2 \operatorname{Sinh} \sqrt{S_{c} R_{c}} h}+\frac{S_{c} S_{r} E_{c} G N^{2} A_{1}^{2}}{2}\left(\frac{\operatorname{Cosh} 2 N h}{4 N^{2}-S_{c} R_{c}}+\frac{1}{S_{c} R_{c}}\right)\right) .
\end{gathered}
$$

\section{Results and Discussion}

In order to obtain the physical insight of the problem, velocity, temperature and concentration are computed numerically for different values of the emerging parameters, viz., Darcy number is $D_{a}, M$ is the Hartman number, $P_{r}$ is the Prandtl number, $E_{c}$ is the Eckert number and $R_{n}$ is the Radiation parameter using Mathematica and are presented in Figures 2-10. 


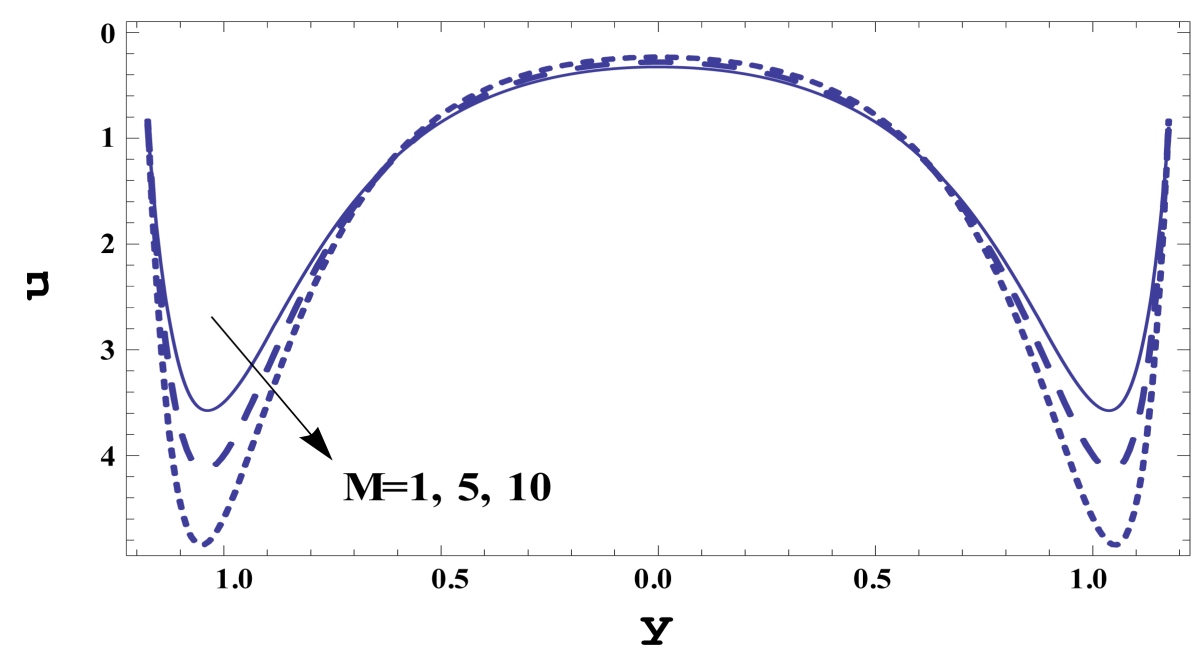

Figure 2. Velocity profiles $u(y)$ for varying values of $M$.

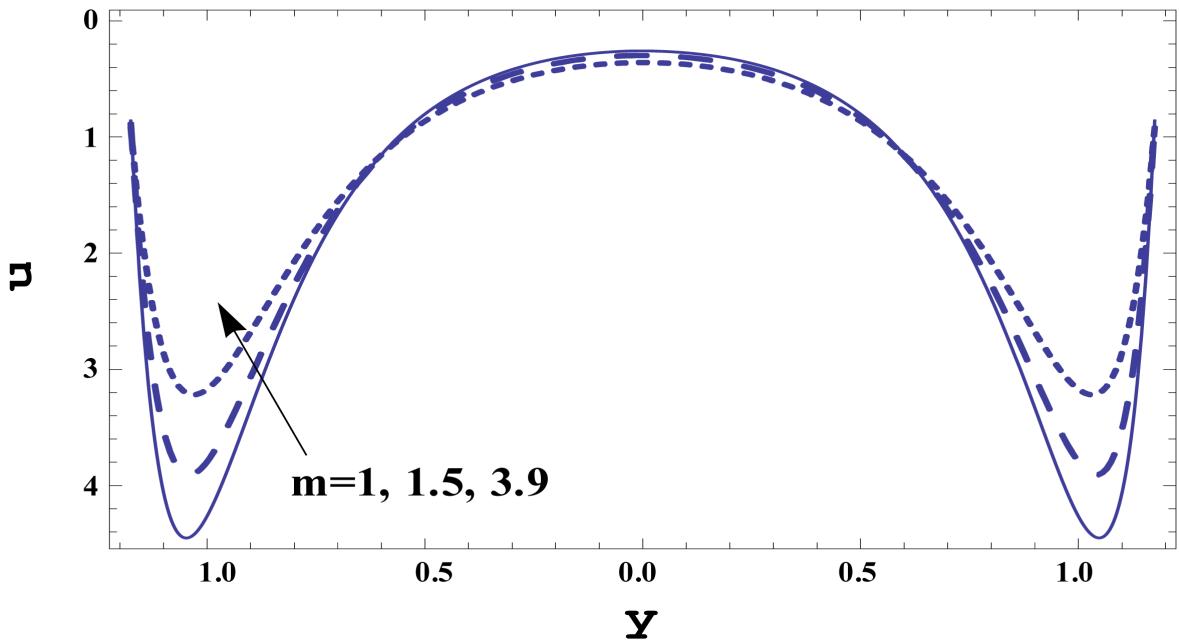

Figure 3. Velocity profiles $u(y)$ for varying values of $m$.

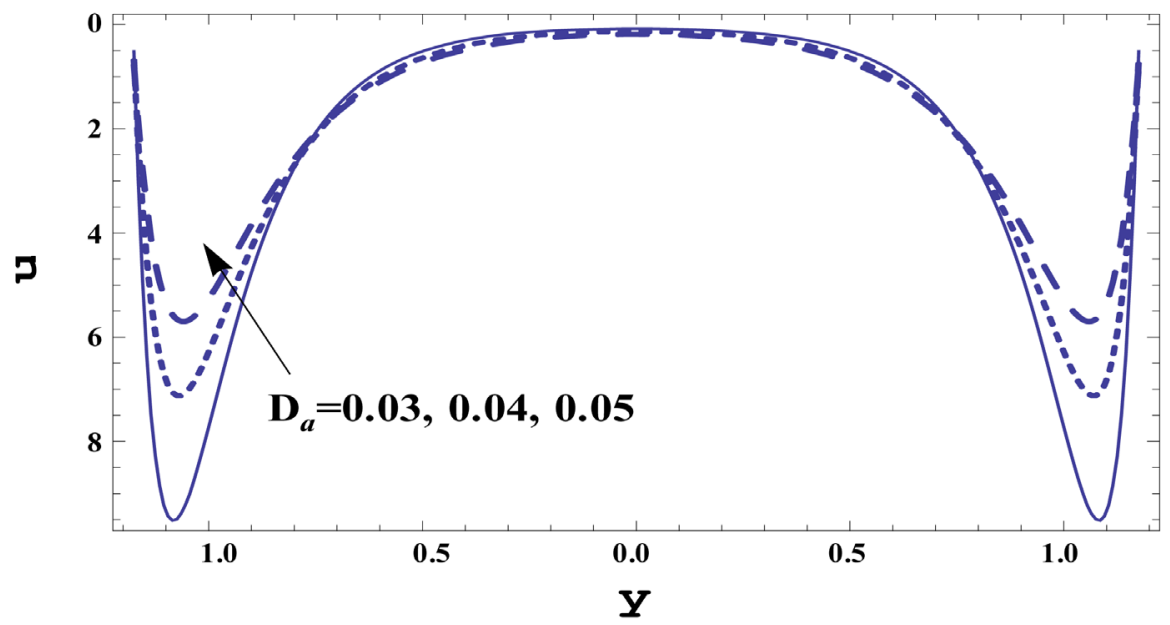

Figure 4. Velocity profiles $u(y)$ for varying values of $D_{a}$. 


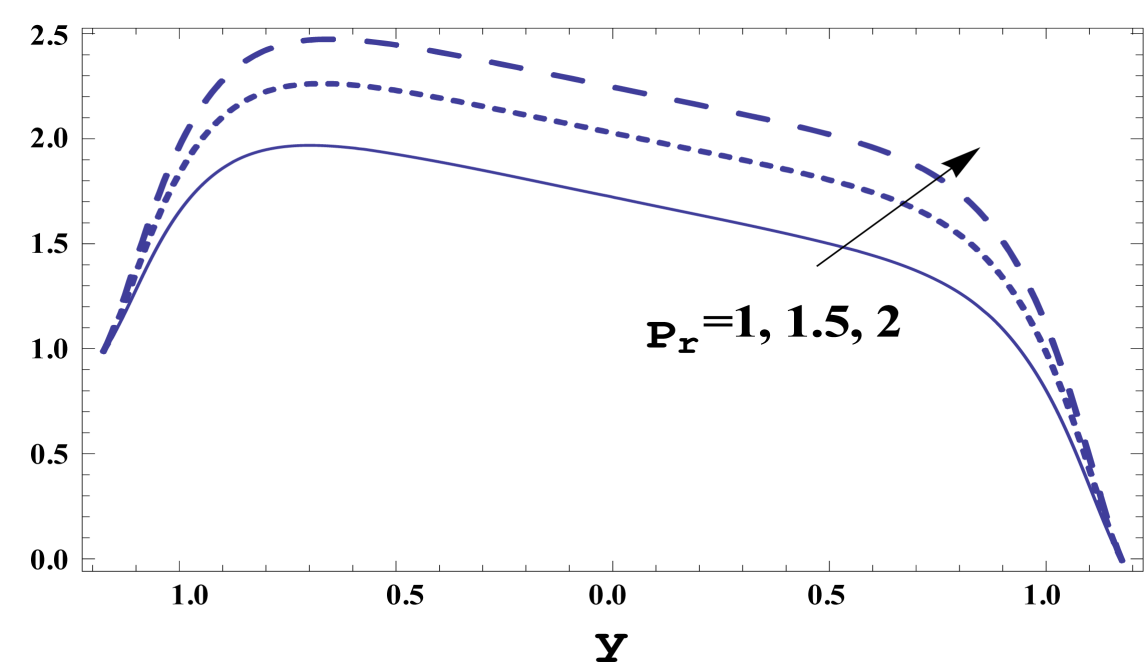

Figure 5. Temperature profiles $\theta(y)$ for varying values of $P_{r}$.

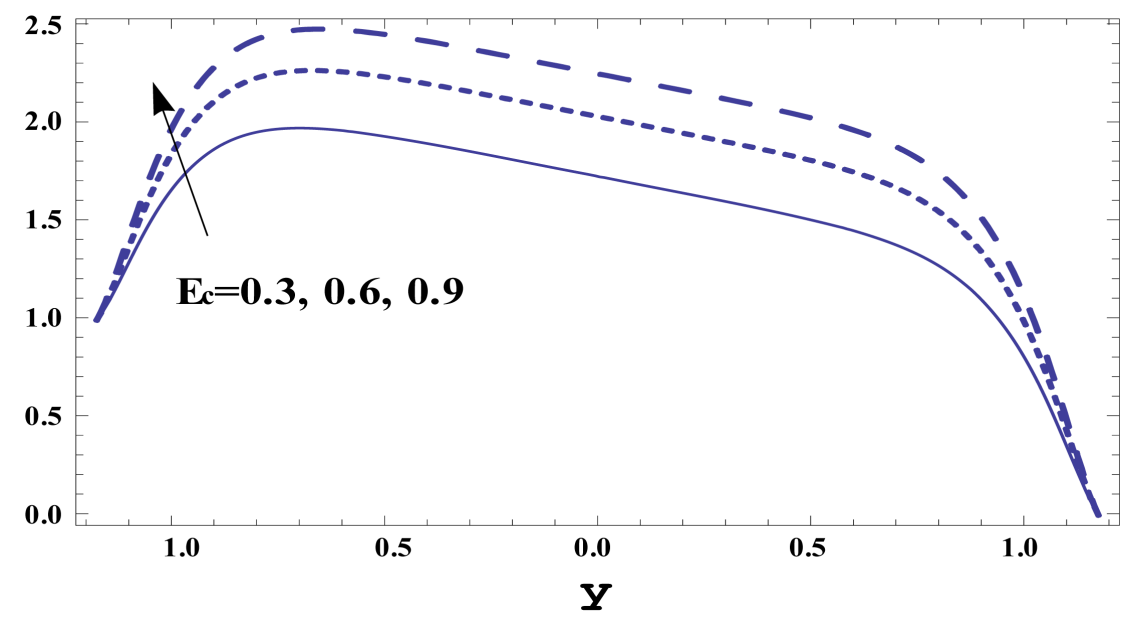

Figure 6. Temperature profiles $\theta(y)$ for varying values of $E_{c}$.

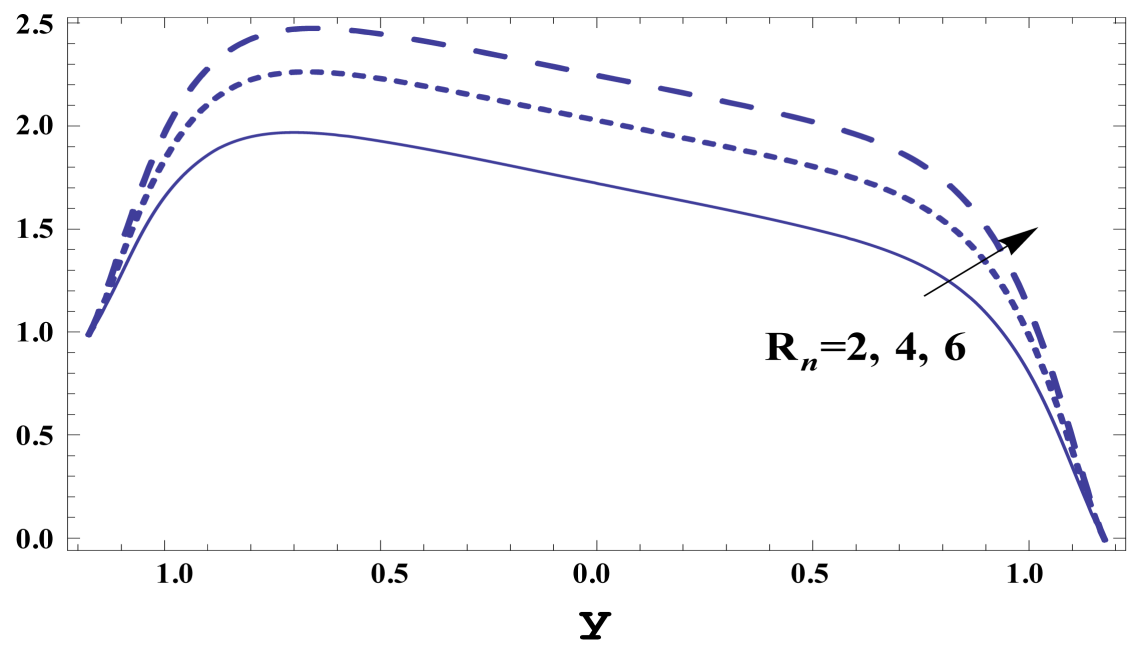

Figure 7. Temperature profiles $\theta(y)$ for varying values of $R_{n}$. 


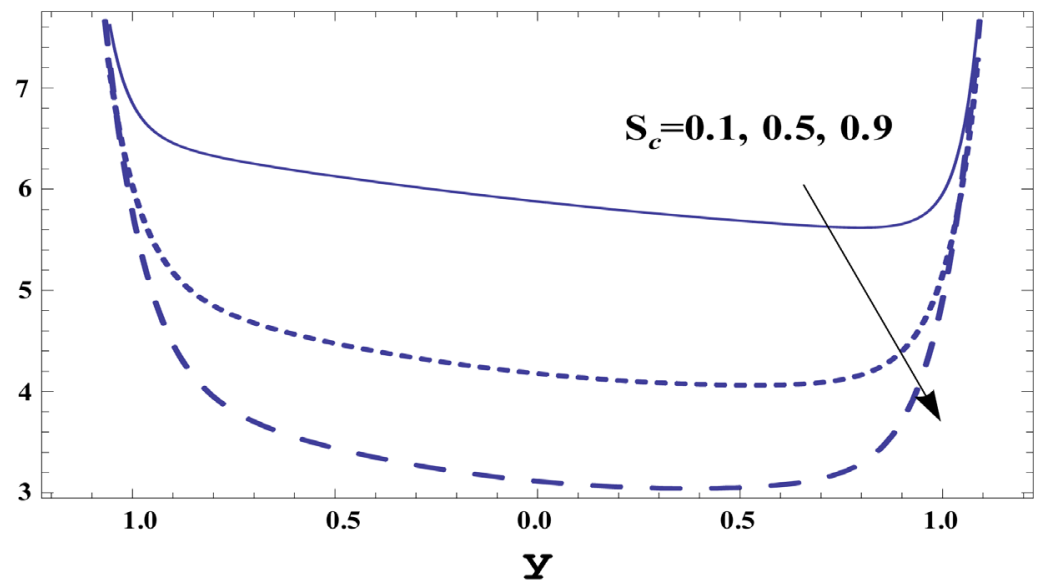

Figure 8. Concentration profiles $\phi(y)$ for varying values of $R_{n}$.

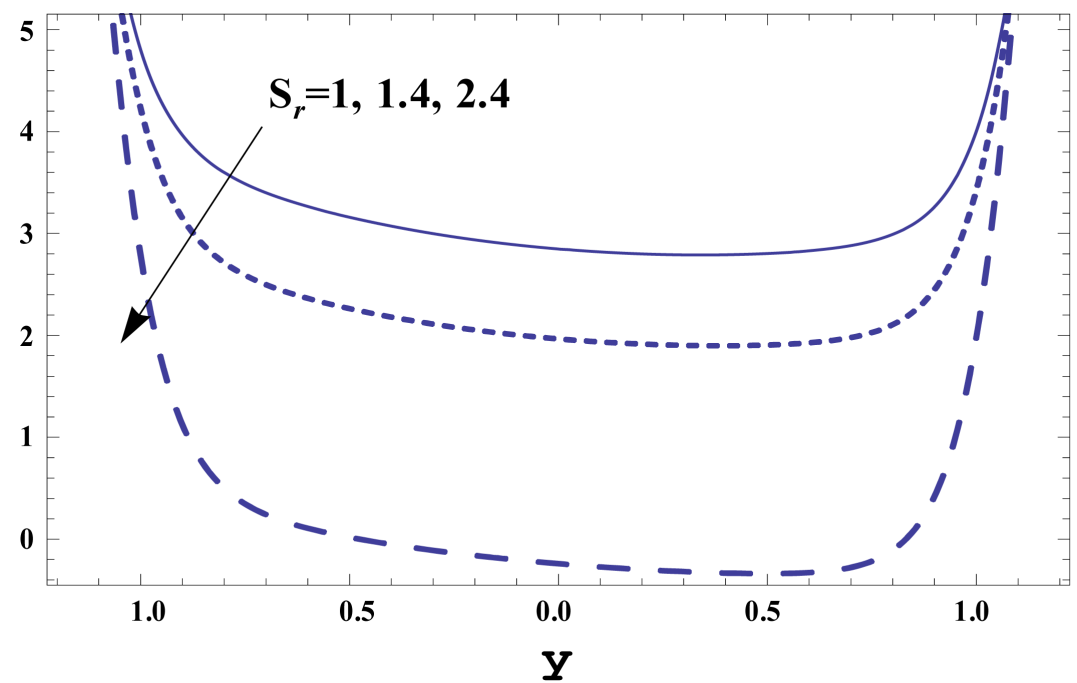

Figure 9. Concentration profiles $\phi(y)$ for varying values of $S_{r}$.

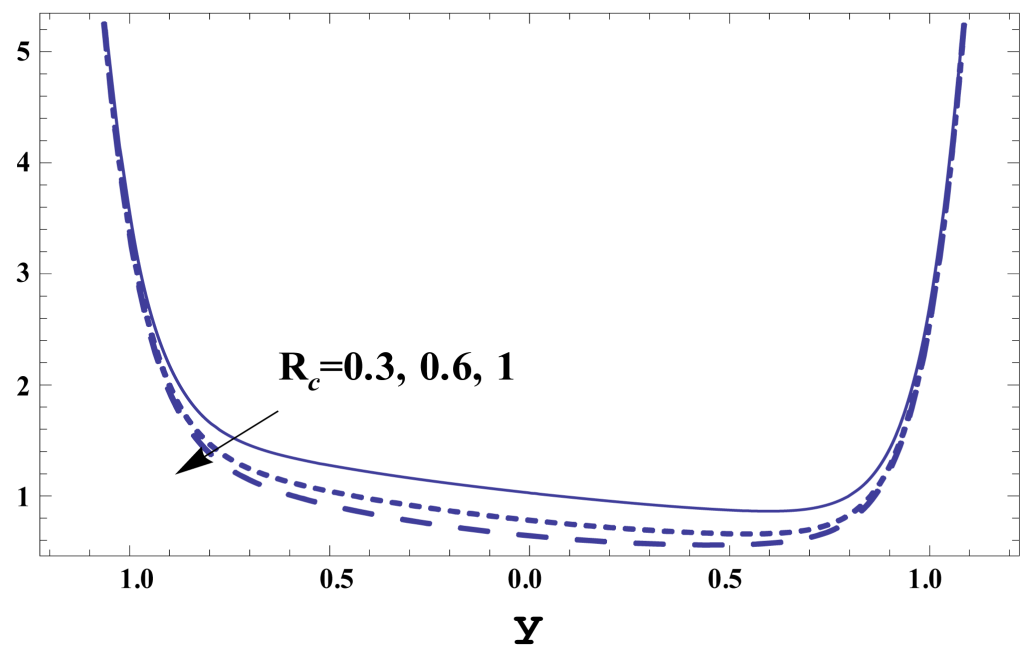

Figure 10. Concentration profiles $\phi(y)$ for varying values of $R_{c}$. 
Figure 2 presents the effect of Hartman number $M$ on the velocity. It is noted that the velocity increases by increasing the Hartman number in the interval $[-0.6,0.6]$ and vice versa in the other intervals.

Figure 3 shows the effect of the Hall parameter $m$ on the velocity. It is observed that as $m$ increases the velocity decreases in the interval $[-0.6,0.6]$ and vice versa in the other intervals.

Figure 4 shows the effect of Darcy parameter $D_{a}$ against the velocity. It is found that the velocity decreases by the increasing of $D_{a}$ in the interval $[-0.6,0.6]$ and vice versa in the other intervals.

Figures 5-7 describe the effect of different parameters on the temperature distribution $\theta$. It is found that the temperature increases as the Prandtle number $P_{r}$ increases this is shown in Figure 5, also in Figure 6 it is observed that the temperature increases as the Eckert number $E_{c}$ increases. In Figure 7 the temperature increases as the Radiation parameter $R_{n}$ increases.

Figures 8-10 display results for the concentration $\phi$ profiles. It is clear that the concentration decreases as the Schmidt number $S_{c}$ increases this is shown in Figure 8, also in Figure 9 and Figure 10 the concentration decreases as the Soret number $S_{r}$, Chemical reaction parameter $R_{C}$ respectively.

\section{Conclusions}

In this paper, we studied the effects of the physical parameters of the considered problem on peristaltic transport in a tube, filled with an incompressible non-Newtonian (Third order) fluid, and considered the effects of hall current, body temperature and concentration. The system is solved analytically by perturbation technique. The effects of various emerging parameters on the flow, the temperature and the concentration distributions are shown and discussed with the help of graphs. The main findings can be summarized as follows.

1) The velocity decreases in the interval $[-0.6,0.6]$ and vice versa in the other intervals with the increase of each of $m$ and $D_{a}$, whereas it increases as $M$ increase.

2) The temperature $T$ increases with the increase of each of as the Prandtle number $P_{r}$, the Eckert number and the Radiation parameter $R_{n}$.

3) The concentration decreases as the Schmidt number $S_{c}$, the Soret number $S_{r}$ and Chemical reaction parameter $R_{C}$ increases.

\section{Caption of Figures}

Figure 2 the velocity profiles are plotted versus $y$ for different values of $M$ for a system have the particulars $\phi=0.2, m=1, D_{a}=0.08, x=\frac{\pi}{3}$.

Figure 3 the velocity profiles are plotted versus $y$ for different values of $m$ for a system have the particulars $\phi=0.2, M=10, D_{a}=0.09, x=\frac{\pi}{3}$.

Figure 4 the velocity profiles are plotted versus $y$ for different values of $D_{a}$ for a system have the particulars $M=1, \phi=0.2, m=1, x=\frac{\pi}{3}$.

Figure 5 the temperature profiles are plotted versus $y$ for different values of $P_{r}$ for a system have the particulars $M=5, \phi=0.2, m=1, D_{a}=0.08, x=\frac{\pi}{3}, E_{c}=2$.

Figure 6 the temperature profiles are plotted versus $y$ for different values of $E_{c}$ for a system have the particulars $M=5, \phi=0.2, m=1, D_{a}=0.08, x=\frac{\pi}{3}, P_{r}=1, R_{n}=2$.

Figure 7 the temperature profiles are plotted versus $y$ for different values of $R_{n}$ for a system have the particulars $M=5, \phi=0.2, m=1, D_{a}=0.08, x=\frac{\pi}{3}, P_{r}=1, E_{c}=0.3$.

Figure 8 The concentration profiles are plotted versus $y$ for different values of $S_{c}$ for a system have the particulars $M=5, \phi=0.2, m=1, D_{a}=0.08, x=\frac{\pi}{3}, P_{r}=1.5, E_{c}=0.9, R_{n}=2, S_{r}=1, R_{c}=1$. 
Figure 9 the concentration profiles are plotted versus $y$ for different values of $S_{r}$ for a system have the particulars $M=5, \phi=0.2, m=1, D_{a}=0.03, x=\frac{\pi}{3}, P_{r}=1.5, E_{c}=0.9, R_{n}=2, S_{c}=1, R_{c}=1$.

Figure 10 the concentration profiles are plotted versus $y$ for different values of $R_{c}$ for a system have the particulars $M=5, \phi=0.2, m=1, D_{a}=0.03, x=\frac{\pi}{3}, P_{r}=1.5, E_{c}=0.9, R_{n}=2, S_{c}=1, S_{r}=2$.

\section{References}

[1] Latham, T.W. (1966) Fluid Motions in a Peristaltic Pump, M.S. Thesis, M.I.T., Cambridge.

[2] Haroun, M.H. (2007) Effect of Deborah Number and Phase Difference on Peristaltic Transport of a Third-Order Fluid in an Asymmetric Channel. Communications in Nonlinear Science and Numerical Simulation, 12, 1464-1480. http://dx.doi.org/10.1016/j.cnsns.2006.03.002

[3] Eldabe, N.T.M., El-Sayed, M.F., Ghaly, A.Y. and Sayed, H.M. (2007) Peristaltically Induced Transport of a MHD Biviscosity Fluid in a Non-Uniform Tube. Physica A, 383, 253-266. http://dx.doi.org/10.1016/j.physa.2007.05.027

[4] Hayat, T., Afsar, A., Khan, M. and Asghar, S. (2007) Peristaltic Transport of a Third Order Fluid under the Effect of a Magnetic Field. Computers and Mathematics with Applications, 53, 1074-1087. http://dx.doi.org/10.1016/j.camwa.2006.12.014

[5] Srinivas, S. and Kothandapani, M. (2009) The Influence of Heat and Mass Transfer on MHD Peristaltic Flow through a Porous Space with Compliant Walls. Applied Mathematics and Computation, 213, 197-208. http://dx.doi.org/10.1016/j.amc.2009.02.054

[6] Siddiqui, A.M., Rana, M.A. and Ahmed, N. (2008) Effects of Hall Current and Heat Transfer on MHD Flow of a Burgers' Fluid Due to a Pull of Eccentric Rotating Disks. Communications in Nonlinear Science and Numerical Simulation, 13, 1554-1570. http://dx.doi.org/10.1016/j.cnsns.2006.10.005

[7] Hayat, T., Abbas, Z. and Asghar, S. (2008) Effects of Hall Current and Heat Transfer on Rotating Flow of a Second Grade Fluid through a Porous Medium. Communications in Nonlinear Science and Numerical Simulation, 13, 21772192. http://dx.doi.org/10.1016/j.cnsns.2007.03.033

[8] Nowar, K. (2014) Peristaltic Flow of a Nanofluid under the Effect of Hall Current and Porous Medium. Mathematical Problems in Engineering, 2014, 1-15. http://dx.doi.org/10.1155/2014/389581

[9] Eldabe, N.T.M., Fouad, A. and Hussein, M.M. (2010) Magnetohydrodynamic Flow and Heat Transfer for a Peristaltic Motion of Carreau Fluid through a Porous Medium. Journal of Mathematics, 42, 1-16.

[10] Eldabe, N.T.M., Agoor, M. and Alame, H. (2014) Peristaltic Motion of Non-Newtonian Fluid with Heat and Mass Transfer through a Porous Medium in Channel under Uniform Magnetic Field. Journal of Fluids, 2014, 1-12. http://dx.doi.org/10.1155/2014/525769

[11] Eldabe, N.T.M., Sallam, N.S., Mohamed, M.A.A, Abo Zaid, M.Y. and Abd-Elmonem, A. (2011) Magnetohydrodynamic Peristaltic Motion with Heat and Mass Transfer of a Jeffery Fluid in a Tube through Porous Medium. Innovative Systems Design and Engineering, 264-275. 